\title{
CARACTERÍSTICAS FÍSICO-QUÍMICAS DEAMOSTRAS DE MÉIS DE Apis mellifera L. DO ESTADO DO CEARÁ, BRASIL
}

\author{
Physicochemical characteristics of Apis mellifera L. honey samples from the state of Ceará, Brazil
}

\author{
Augusta Carolina de Camargo Carmello Moreti ${ }^{1}$, Geni da Silva Sodréé, Luís Carlos Marchini ${ }^{3}$, Ivani Pozar Otsuk ${ }^{4}$
}

\section{RESUMO}

Objetivou-se, na presente pesquisa, desenvolvida com amostras de méis de Apis mellifera coletadas diretamente dos produtores de diversos municípios do estado de Ceará, Brasil, verificar, com base em características físico-químicas, como se agrupam as amostras de méis. Dentre as 52 amostras de méis analisadas as que continham pólen de Serjania sp. como dominante ou acessório, formam um subgrupo distinto quanto aos caracteres físico-químicos, o que confirma que a origem floral interfere decisivamente nas características dos méis. Pela análise dos componentes principais, pôde-se verificar que os caracteres que mais influenciaram no agrupamento das amostras de méis foram acidez e índice de formol no eixo $\mathrm{X}$ e $\mathrm{pH}$ e cinzas no eixo $\mathrm{Y}$.

Termos para indexação: Análise físico-química, tipo polínico, origem floral, legislação brasileira.

\begin{abstract}
This paper was carried out to determine how the honey samples of the State of Ceará, Brazil may be grouped, based on the physicochemical composition of the honey. The samples were collected in different municipalities. Among the 52 honey samples analyzed it was observed that the samples which have Sejania sp. as dominant or accessory pollen form a different sub-group based on the physicochemical parameters. The results confirm that the honey bee composition is influenced by the plant nectar. The grouping of the honey samples was mostly influenced by acidity and formol index on the $\mathrm{X}$ axis, and $\mathrm{pH}$ and ashes, on the $\mathrm{Y}$ axis.
\end{abstract}

Index terms: Physicochemical analysis, pollen type, floral origin, brazilian legislation

(Recebido em 11de junho de 2007 e aprovado em 20 de fevereiro de 2008)

\section{INTRODUÇÃO}

O conhecimento da origem floral dos méis é muito importante para a caracterização do produto, assim, a análise polínica é um importante instrumento para o reconhecimento das plantas apícolas utilizadas pelas abelhas, como suprimento de néctar e pólen (BARTH, 1989).

Vários parâmetros físico-químicos, como umidade, HMF, açúcares, índice diastásico, condutividade elétrica, viscosidade, proteínas, índice de formol, cinzas, $\mathrm{pH}$, acidez, entre outros, vêm sendo estudados por Almeida-Anacleto \& Marchini (2004), Araújo et al. (2006), Arruda et al. (2005), Azeredo \& Azeredo (1999), Cano et al. (1992, 2001), Costa et al. (1999), Evangelista-Rodrigues et al. (2005), Marchini et al. (2004a,b, 2005), Silva et al. (2004), Sodré et al. (2003, 2005) e Vieira et al. (2005), entre outros, com o objetivo de caracterizar o produto brasileiro o que é uma tarefa bastante difícil exatamente pela diversidade dos méis produzidos em diferentes regiões do Brasil.
Objetivou-se, na presente pesquisa determinar as características físico-químicas de amostras de méis de Apis mellifera de diversos municípios do Estado do Ceará, pesquisando ainda a análise polínica das mesmas e se os parâmetros estudados encontram-se dentro das normas vigentes na legislação brasileira, colaborando para a melhoria de qualidade do produto.

\section{MATERIALE MÉTODOS}

As amostras de méis (num total de 52) foram obtidas diretamente de apicultores dos municípios de Araripe, Santana do Cariri, Assaré, Iguatu, Crato, Missão Velha e Pacajús, no estado do Ceará. As coletas foram realizadas de novembro de 2001 a agosto de 2002 e as amostras acondicionadas em frascos de vidro transparente, hermeticamente fechados, devidamente etiquetadas com as informações do local de produção.

\footnotetext{
${ }^{1}$ Engenheira Agrônoma, Doutora - Instituto de Zootecnia/IZ - Centro de Pesquisa e Desenvolvimento em Zootecnia Diversificada - Rua Heitor Penteado, 56 - Centro - Cx. P. 60 - 13460-000 - Nova Odessa, SP - acmoreti@iz.sp.gov.br

2 Engenheira Agrônoma, Doutora - Universidade Federal do Recôncavo Baiano/UFRB - Grupo Insecta - Centro de Ciências Agrárias, Ambientais e Biológicas - Campus Universitário, Laboratório de Entomologia - Cx. P. 118 - 44380-000 - Cruz das Almas, BA - gssodre@yahoo.com.br ${ }^{3}$ Engenheiro Agrônomo, PhD - Professor do Departamento de Entomologia, Fitopatologia e Zoologia Agrícola - Escola Superior de Agricultura "Luiz de Queiroz"/ESALQ/USP - Avenida Pádua Dias, 11 - Cx. P. 09 - 13418-900 - Piracicaba, SP - Icmarchi@esalq.usp.br - Bolsista de Produtividade em Pesquisa do CNPq.

${ }^{4}$ Engenheira Agrônoma, Mestre - Instituto de Zootecnia/IZ - Centro de Pesquisa e Desenvolvimento de Genética e Reprodução Animal - Rua Heitor Penteado, 56 - Centro - Cx. P. 60 - 13460-000 - Nova Odessa, SP - ivani@iz.sp.gov.br
} 
As análises físico-químicas e polínicas foram realizadas no Laboratório de Apicultura do Departamento de Entomologia, Fitopatologia e Zoologia Agrícola da Escola Superior de Agricultura "Luiz de Queiroz", campus de Piracicaba, da Universidade de São Paulo, assim que as amostras foram recebidas.

As seguintes análises físico-químicas foram realizadas em triplicata: açúcares redutores, redutores totais e sacarose - Copersucar (1987) modificado por Marchini et al. (2004a); umidade (ATAGO, 1988); hidroximetilfurfural (HMF) (AOAC, 1990); cor (VIDAL \& FREGOSI, 1984); condutividade elétrica (BOLETIN OFICIAL ESPAÑOL, 1986); pH, acidez e índice de formol (MORAES \& TEIXEIRA, 1998); teor de cinzas (PREGNOLATO \& PREGNOLATO, 1985); proteínas (SILVA \& QUEIROZ, 2002), viscosidade (CAMPOS, 1989) e atividade diastásica (BRASIL, 2000).

Para análise polínica as amostras foram submetidas ao método da acetólise (ERDTMAN, 1952) e em seguida realizadas uma análise qualitativa, por meio da identificação por comparação dos tipos polínicos presentes nas amostras (BARTH, 1970a,b,c, 1989) e outra quantitativa, por meio da contagem sucessiva de 300 grãos de pólen, classificandose os tipos polínicos em classes de ocorrência conforme Louveaux et al. (1978).

Os dados foram analisados por meio da análise multivariada, utilizando-se os componentes principais para avaliar a importância de cada caracter físico-químico estudado sobre a variação total disponível (MARDIA et al., 1979).

Para a análise de agrupamento, adotou-se segundo Bussab et al. (1990), como média de dissimilaridade a distância euclidiana média para os dados devidamente padronizados. Os agrupamentos foram formados pelo método UPGA (unweighted pair-group average).

\section{RESULTADOS E DISCUSSÃO}

Os resultados obtidos são apresentados na Tabela 1.

A cor está correlacionada com a sua origem floral, processamento e armazenamento, fatores climáticos durante o fluxo do néctar e com a temperatura na qual o mel se forma no interior da colméia (SEEMANN \& NEIRA, 1988).

Observa-se que as amostras do estado do Ceará foram bem mais claras do que as que vêm sendo observadas para os méis brasileiros de uma maneira geral (MORETI et al., 2006). Foram classificadas como branco-água, 26,92\% das amostras; como branco, $17,31 \%$ e como extra-branco, $17,31 \%$; âmbarextra-claro (11,54\%), âmbar (9,61\%), âmbar-claro (15, 38\%) e âmbar-escuro $(1,92 \%)$ As cores das amostras estão dentro dos limites estabelecidos pela norma vigente que pode variar desde o branco-água até âmbar-escuro (BRASIL, 2000).
A umidade (\%) variou de 15,00 a $20,3 \%$, com uma média de 17,4 (Tabela 1), estando dentro do limite estabelecidos pela norma vigente que é de no máximo $20 \%$.

Somente duas amostras (36 e 38), representando $3,8 \%$ do total de amostras, ultrapassaram o limite máximo permitido pela legislação, desclassificando-as como mel de mesa. Provavelmente, os méis tenham sido colhidos antes da operculação total dos favos, o que explicaria a alta umidade do produto final.

Cano et al. (1992) observaram $24,1 \%$ do total de amostras acima dos valores permitidos pela legislação, em amostras de méis consumidos em São Paulo, o que indica um manejo adequado por parte dos apicultores.

O valor médio obtido (17,4\% de umidade) está próximo das médias verificadas por Costa et al. (1999) quando estudaram méis de diferentes regiões do Brasil.

Cano et al. (2001) comparando amostras de méis brasileiros de flores de eucalipto e de laranjeira, verificaram que a umidade variou de 16,9 a $18,4 \%$ para eucalipto e 15,5 a $16,8 \%$ para os de flores de laranjeira; valores esses próximos aos obtidos por Vilhena \& Almeida-Muradian (1999) para méis de São Paulo e aos obtidos no presente trabalho, para méis do estado do Ceará.

Os valores de condutividade elétrica variaram de 154,7 a 1667,7 $\mu \mathrm{S}$, com média de 383,0 $\mu \mathrm{S}$ (Tabela 1 ), que é inferior à 448,6 $\mu \mathrm{S} \mathrm{cm}^{-1}$, encontrada por Gomez et al. (1993), para amostras de méis comerciais de eucaliptos da Espanha e de $568,25 \mu \mathrm{S} \mathrm{cm}^{-1}$ observada para méis silvestres.

No Brasil, vários autores têm verificado valores bastante variáveis para condutividade elétrica, como Sodré et al. (2002) que obtiveram valores variando de 271,67 a $1634,00 \mu \mathrm{S} . \mathrm{cm}^{-1}$, com média de $780,70 \mu \mathrm{S} . \mathrm{cm}^{-1}$ para méis da região litoral norte do estado da Bahia, Almeida-Anacleto $\&$ Marchini (2004) que constataram 793,56 $\mu$ S.cm-1 para amostras de mel do cerrado paulista, Marchini et al. (2005) $568,25 \mu \mathrm{S} . \mathrm{cm}^{-1}$ para mel de flores de laranjeira e de 1018,65 para mel de eucalipto, Vieira et al. (2005) 1096,00 $\mu{\mathrm{S} . \mathrm{cm}^{-1}}^{-1}$ para amostras de méis de cerrado do Mato Grosso e Arruda et al. (2005), apenas $205 \mu \mathrm{S} . \mathrm{cm}^{-1}$ para amostras de méis da Chapada do Araripe, CE.

$\mathrm{O}$ valor médio obtido para a condutividade elétrica encontra-se dentro dos limites estabelecidos pela norma internacional (BOGDANOV et al., 1997) que é de 200 a $800 \mu \mathrm{S}$, no entanto, foram observadas nove amostras $(3,4,5,8,9,11,13,18$ e 23$)$ com valores abaixo do estabelecido e mais duas amostras (44 e 52) com valores acima do limite superior. Isso pode ser explicado porque existe uma correlação entre condutividade elétrica e origem floral (AGANIN, 1971) e os valores fixados como limites, superior e inferior, foram baseados em méis de origem botânica diferente dos estudados no presente trabalho. 


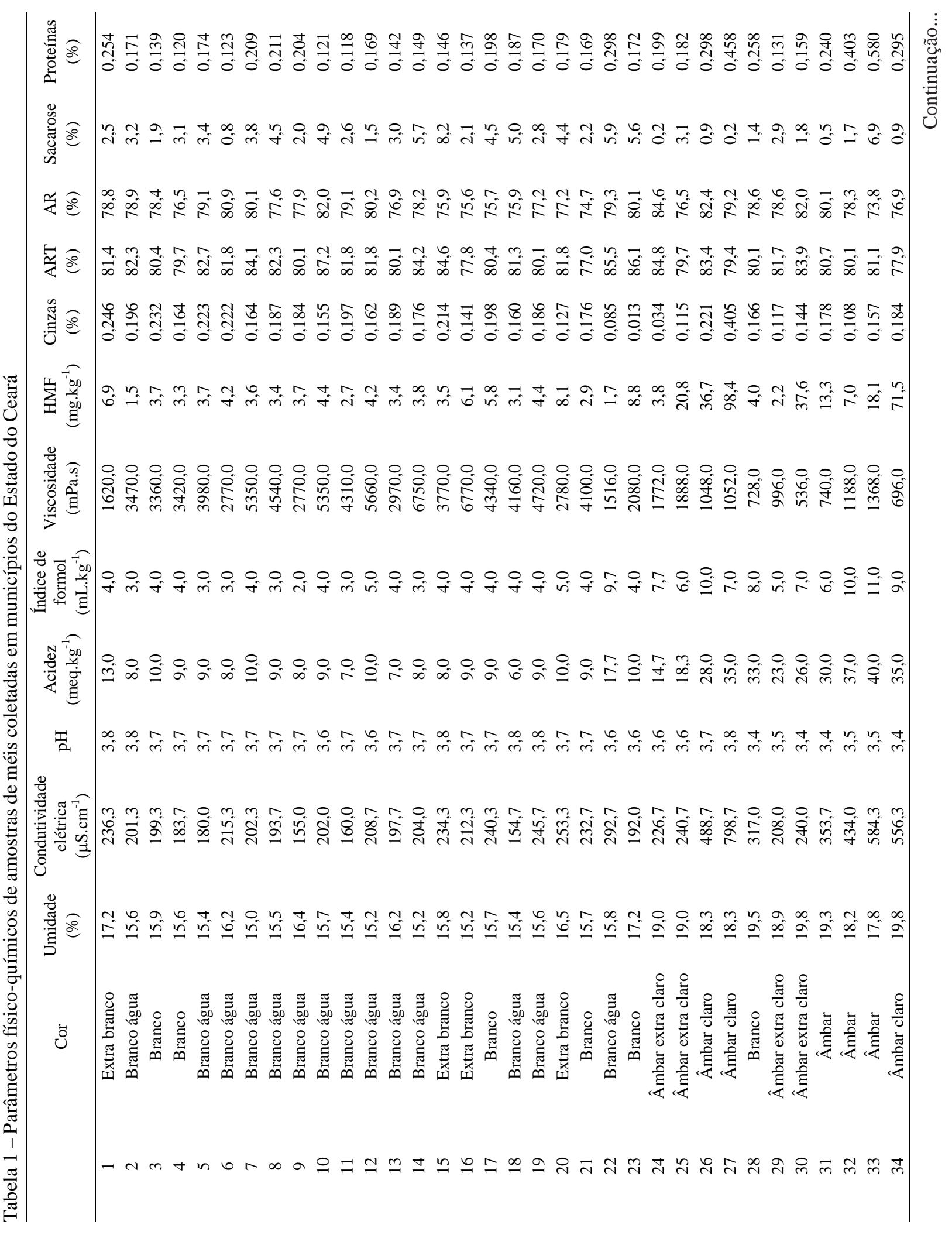

Ciênc. agrotec., Lavras, v. 33, n. 1, p. 191-199, jan./fev., 2009 


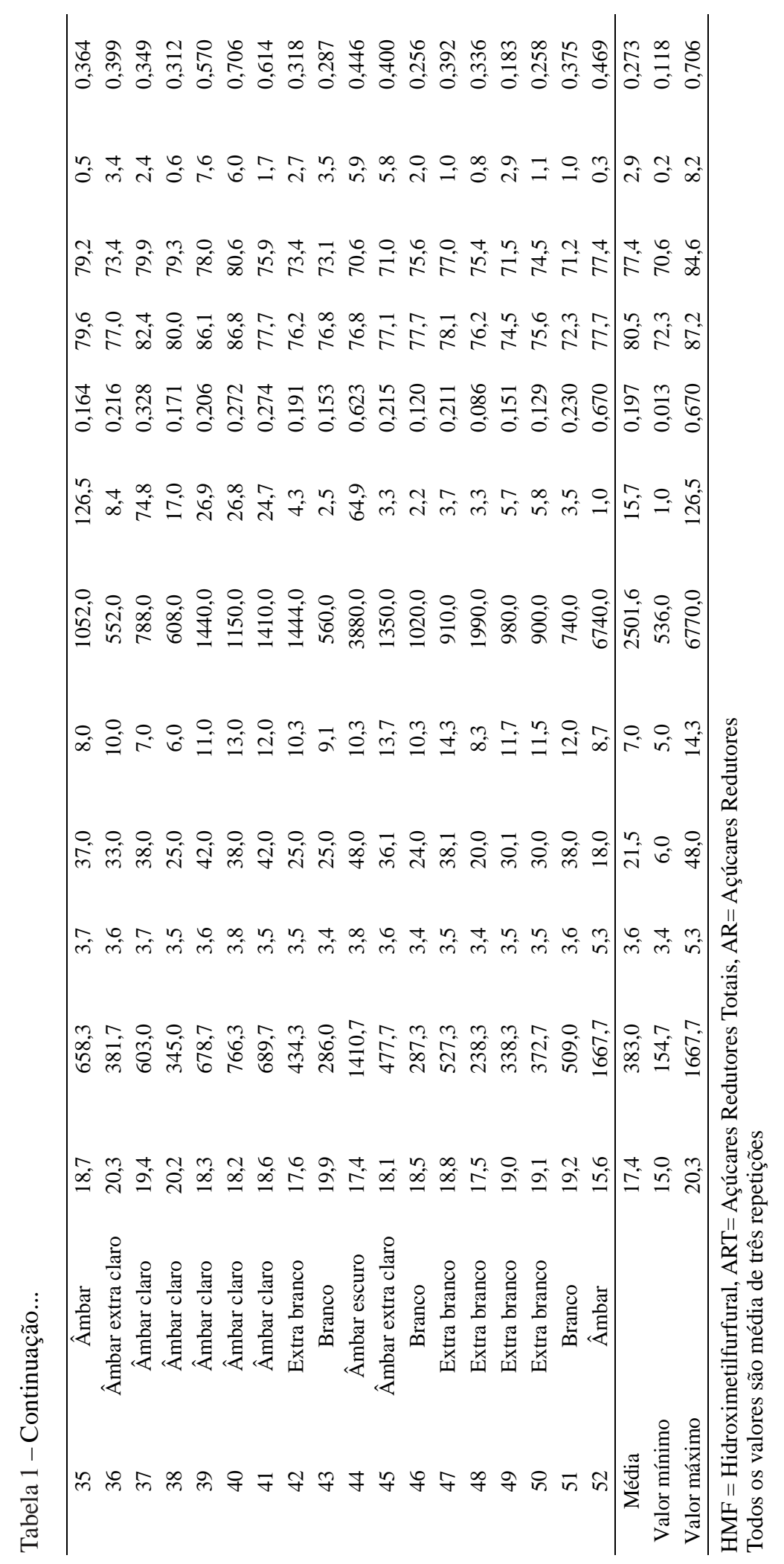

Ciênc. agrotec., Lavras, v. 33, n. 1, p. 191-199, jan./fev., 2009 
$\mathrm{O} \mathrm{pH}$ apresentou valores variando de 3,4 a 5,3, com valor médio de 3,6 (Tabela 1). Apenas uma amostra (52) teve valor de $\mathrm{pH}$ acima do limite superior estabelecidos pela norma vigente que é de 4,6 .

Os valores encontrados são bastante próximos aos obtidos por Araújo et al. (2006), Azeredo \& Azeredo (1999), Evangelista-Rodrigues et al. (2005), Silva et al. (2004) e Sodré et al. (2002), em amostras de méis de diferentes regiões brasileiras.

A acidez apresentou valores variando de 6,0 a 48,0 meq kg-1 (média de 21,5meq kg-1)(Tabela 1), estando assim, em conformidade com o valor estabelecido pela legislação vigente que é, de no máximo, $50 \mathrm{meq} \mathrm{kg}^{-1}$. Esse valor é inferior ao obtido por Araújo et al. (2006), Evangelista-Rodrigues (2005) e Sodré et al. (2002), mas bem próximo à média verificada por Costa et al. (1999) e Silva et al. (2004), em amostras de méis de diferentes regiões brasileiras.

Os valores para o índice de formol variaram 5,0 a $14,3 \mathrm{~mL} \mathrm{~kg}^{-1}$, com um valor médio de $7,0 \mathrm{~mL} \mathrm{~kg}^{-1}$ (Tabela 1), próximo ao verificado por Sodré et al. (2002) e pouco inferior ao observado por Almeida-Anacleto \& Marchini (2004), Marchini et al. (2004b) e Vieira et al. (2005). A totalidade das amostras está dentro dos limites estabelecidos pela norma vigente que é de 4,5 a $15 \mathrm{~mL} \mathrm{~kg}^{-1}$.

Os valores de viscosidade (mPa.s) encontrados nas amostras de méis analisadas, variaram 356,0 a 67702,0 com valor médio de 2501,6 na temperatura de $20^{\circ} \mathrm{C}$ (Tabela 1$)$.

Sodré et al. (2002) encontraram uma média de 1607 $\mathrm{mPa}$.s para viscosidade nos méis provenientes dos estados do Ceará, Piauí e Pernambuco. Enquanto em amostras de méis de laranjeira provenientes do Estado de São Paulo, Marchini et al. (2004b) observaram uma média de 1096,00 $\mathrm{mPa}$.s a qual é pouco inferior aos valores verificados no presente trabalho, enquanto Arruda et al. (2005) verificaram valor médio muito superior (4140,95 mPa.s).

As quantidades de hidroximetilfurfural (HMF) variaram de 1,0 a 126,5 $\mathrm{mg} \mathrm{kg}^{-1}$ com valor médio de $15,7 \mathrm{mg}$ $\mathrm{kg}^{-1}$ (Tabela 1). Dentre as amostras analisadas cinco (27, 34, 35, 37 e 44) estão fora do limite estabelecido pela norma vigente que é de no máximo $60 \mathrm{mg} \mathrm{kg}^{-1}$. Existem duas hipóteses para explicar o alto índice de HMF nessas amostras: ou os méis foram aquecidos, o que teria provocado formação do HMF, ou o mel foi armazenado por um tempo mais longo em ambiente com alta temperatura, uma vez que o HMF é um indicador de superaquecimento, armazenamento prolongado ou adulteração com açúcar invertido.

Dayrell \& Vital (1991), analisando amostras de méis brasileiros, constataram valores variando de 1,10 a 248,20 $\mathrm{mg} \mathrm{kg}^{-1}$. Esses autores mencionaram que os méis de países tropicais possuem alto teor de HMF, tornando-se fundamental a quantificação desse componente, para a verificação da qualidade do produto.

Sodré et al. (2002) observaram valores variando de 1,50 a 136,00 mg kg-1, com média de $24,33 \mathrm{mg} \mathrm{kg}^{-1} \mathrm{em}$ amostras de méis da região litoral norte da Bahia, média essa superior à observada para os méis do Ceará, no presente estudo. Em amostras de diferentes regiões brasileiras Costa et al. (1999) observaram média de 14,1 mg $\mathrm{kg}^{-1}$, Almeida-Anacleto \& Marchini (2004) 3,7 mg kg-1, Marchini et al. (2004b) 19,65 $\mathrm{mg} \mathrm{kg}^{-1}$, Arruda et al. (2005) $4,12 \mathrm{mg} \mathrm{kg}^{-1}$, Evangelista-Rodrigues et al. (2005) valores de 20,7 $223,9 \mathrm{mg} \mathrm{kg}^{-1}$, Vieira et al. (2005) média de 2,7 $\mathrm{mg} \mathrm{kg}^{-1} \mathrm{e}$ Araújo et al. (2006) de 2,88 até $340,03 \mathrm{mg} \mathrm{kg}^{-1}$.

Os valores de cinzas variaram 0,013 a $0,6703 \%$ (média de 0,197\%) (Tabela 1), o que está de acordo com a legislação brasileira que estabelece o limite máximo de $0,6 \%$. Apenas duas amostras (44 e 52) ultrapassaram esse limite.

Os valores de cinzas em amostras de méis do estado do Ceará foram próximos à média obtida por Vilhena \& Almeida-Muradian (1999) (0,14\%). Amostras de diferentes regiões brasileiras apresentaram teor de cinzas variável: Silva et al. (2004) verificaram valores variando de 0,06 até 0,4\%; Marchini et al. (2004b) 0,183\%; Almeida-Anacleto \& Marchini (2004) 0,28\%; Evangelista-Rodrigues et al. (2005), de 0,17 a 0,20; Arruda et al. (2005) 0,185\% e Araújo et al. (2006) de 0,06 a $0,15 \%$.

A quantidade de açúcares redutores totais variou de 72,3 a 87,2\%, com valor médio de $80,5 \%$ (Tabela 1 ).

Para os açúcares redutores totais não existe valor estabelecido na norma vigente, mas as médias observadas estão próximas das obtidas por Sodré et al. (2002) e Vilhena \& Almeida-Muradian (1999), em méis de diferentes regiões brasileiras e das de Baldi-Coronel et al. (1993) e Sporns et al. (1992).

O conteúdo de açúcares redutores variou de 70,6 a $84,6 \%$ (média de $77,4 \%$ ), com valores acima do estabelecido como limite mínimo pelas normas vigentes que é de $65 \%$. 
Os valores de sacarose (\%) variaram de 0,2 a $8,2 \%$ (média de $2,9 \%$ ) e não excedem a valor máximo (6\%) permitido pela legislação, entretanto, foram observados em três amostras (15, 33 e 39) (5,8\% do total de amostras) valores acima do estabelecido pela norma vigente (Tabela 1). Provavelmente, a abelha colheu outra fonte de alimento que não néctar para produzir o respectivo mel.

Cano et al. (1992) verificaram que seis $(10,3 \%)$ das 173 amostras de méis comercializados na capital de São Paulo continham valores de sacarose aparente acima dos permitidos pela legislação vigente (BRASIL, 2000), e do observado para as amostras do estado do Ceará $(5,8 \%)$.

O conteúdo de proteína variou de 0,118 a 0,706 (média de $0,273 \%$ ) (Tabela 1), estando próximo dos padrões internacionais (0,26\%), e aos de Sodré et al. (2002).

Em diferentes regiões brasileiras, Marchini et al. (2004b) verificaram teor de proteínas em amostras de mel de 0,344\%, enquanto Almeida-Anacleto \& Marchini (2004), Arruda et al. (2005) e Vieira et al. (2005) obtiveran (0,23\%, $0,166 \%$ e $0,31 \%$, respectivamente).

Para a análise dos componentes principais dos 13 caracteres físico-químicos existentes na Tabela 1 , foram usados nove: cor, umidade, $\mathrm{pH}$, índice de formol, HMF, cinzas, açúcares redutores totais, sacarose e proteína, para a análise de agrupamento e descartados: condutividade elétrica, acidez, viscosidade e açúcares redutores devido à alta correlação apresentada com algum outro parâmetro (JOLIFFE, 1973).

Mardia et al. (1979) afirmaram que, se numa análise de componentes principais os dois ou três primeiros componentes acumularem uma porcentagem relativamente alta da variação total, em geral mais de $70 \%$, eles explicariam a variabilidade manifestada entre variedades avaliadas. No caso presente, observa-se que foram necessários três componentes principais para explicar $76 \%$ da variância total disponível entre os caracteres físico-químicos (Tabela 2). Assim, constata-se uma considerável dispersão da variância no material estudado, razão pela qual optou-se pela análise de Cluster.

No fenograma com os dados da análise de Cluster (Figura 1), pôde-se observar que foram formados cinco grupos identificados a seguir: no grupo I encontram-se as
Tabela 2 - Estimativas das variâncias (autovalores) e porcentagem acumulada da variância total (\%), obtidas através da análise de componentes principais considerando as 52 amostras de mel e nove caracteres físico-químicos.

\begin{tabular}{ccc}
\hline $\begin{array}{c}\text { Componentes } \\
\text { principais }\end{array}$ & Autovalores & \% acumulada \\
\hline $\mathrm{Y}_{1}$ & 5,354 & 41,19 \\
$\mathrm{Y}_{2}$ & 2,774 & 62,52 \\
$\mathrm{Y}_{3}$ & 1,753 & 76,01 \\
\hline
\end{tabular}

amostras 44, 37 e 34, no grupo II a amostra 35, no grupo III a amostra 27, no grupo IV, quatro amostras (40, 39, 30 e 26), no grupo $\mathrm{V}$, cinco amostras $(41,33,38,31$ e 25$)$ e no grupo VI, o maior deles, 38 amostras (52, 36, 32, 47, 45, 51, 50, 49, $46,43,48,42,28,22,23,15,10,29,24,21,16,12,6,9,19,13$, $4,3,14,7,18,8,5,11,2,17,20$ e 1).

Adotou-se como critério para definição dos grupos o gráfico das distâncias de ligação nos sucessivos passos da análise de agrupamento. Esse método baseia-se na identificação de um platô no sentido vertical, o que significa que muitos grupos foram formados na mesma distância de ligação. Essa distância pode ser um ponto ótimo de corte no dendrograma determinando o número de grupos formados. No presente trabalho, o ponto ótimo para corte escolhido foi 16 , representado pela linha tracejada na Figura 1.

Observa-se que todas as amostras que continham o pólen de Serjania sp como dominante ou acessório (22 amostras) agruparam-se, com base nas características físicas e químicas, no Grupo VI (subgrupo II desse grupo) o que indica que a origem floral interfere decisivamente nas características dos méis. A análise de agrupamento já foi usada para estudar amostras de méis do Estado de São Paulo, por Marchini et al. (2005), possibilitando verificar que as amostras de mesma origem floral foram agrupadas nos mesmos grupos ou subgrupos. As demais amostras do presente estudo continham proporções variáveis principalmente de plantas do gênero Mimosa (Mimosa caesalpineafolia, M. verrucosa e M. scabrella).

Pela análise dos componentes principais, pôde-se verificar que os caracteres que mais influenciaram no agrupamento das amostras de méis foram acidez e índice de formol no eixo $\mathrm{X}$ e $\mathrm{pH}$ e cinzas no eixo $\mathrm{Y}$. 


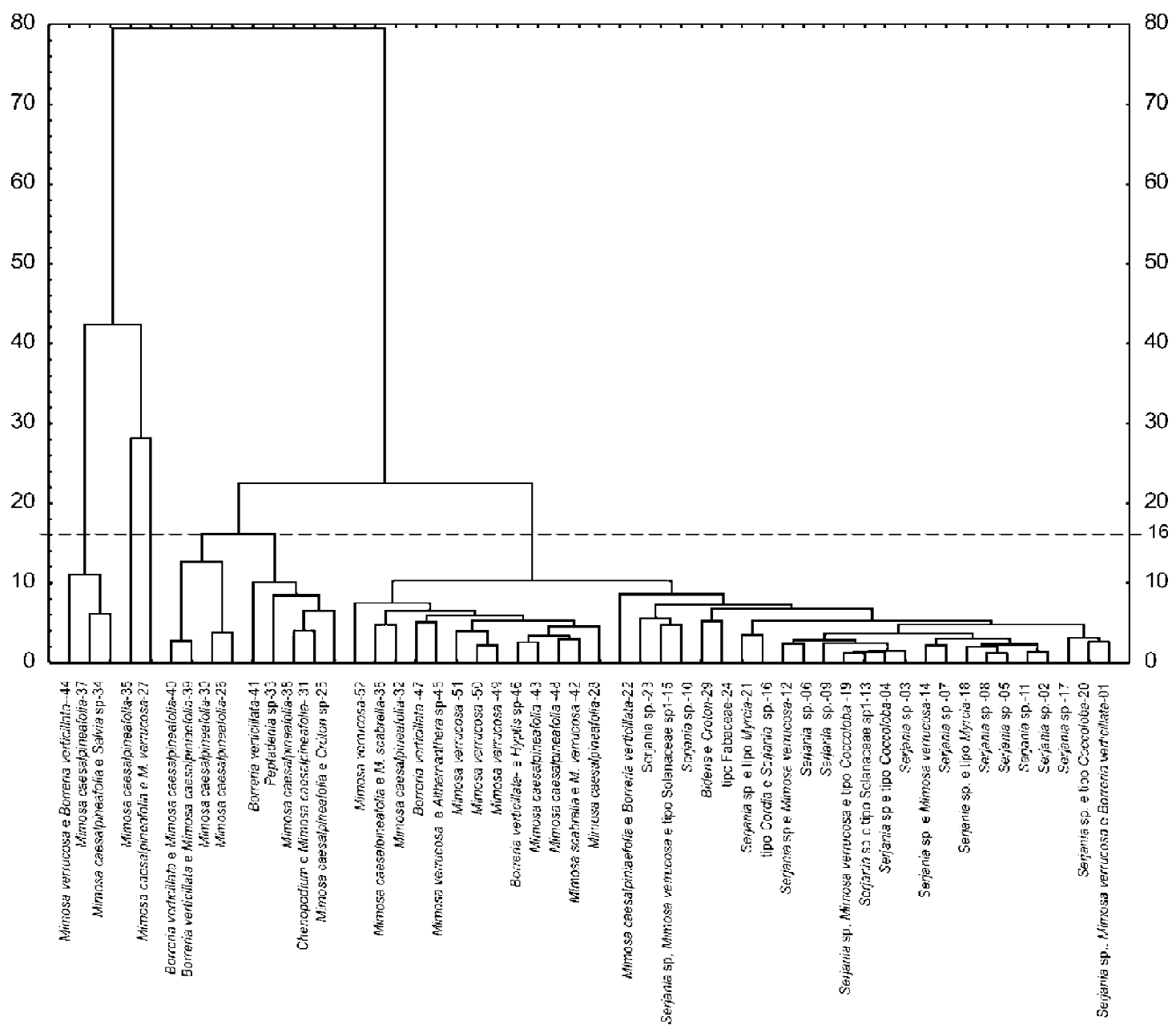

Figura 1 - Fenograma por UPGMA e distância euclidiana média de 52 amostras de méis de Apis mellifera, do estado do Ceará

\section{CONCLUSÕES}

Dentre as amostras de méis do estado do Ceará, as que contêm pólen de Serjania sp., na proporção de pólen dominate ou acessório formam um subgrupo distinto quanto aos caracteres físico-químicos.

A origem floral interfere decisivamente nas características dos méis.

Os valores médios dos parâmetros analisados e que constam da legislação vigente, encontram-se dentro dos limites estabelecidos, indicando um manejo adequado de produção e manipulação do mel analisado.

\section{AGRADECIMENTO}

À FAPESP pelo suporte tecnológico ao trabalho, com fornecimento de equipamentos e regentes, tornando viável a execução do presente trabalho.

\section{REFERÊNCIAS BIBLIOGRÁFICAS}

AGANIN, A. F. Electrical condutivity of several unifloral honeys. Trudy Saratovskogo Zootekhnicheskogo Instituta, Soratov, v. 21, p. 137-144, 1971.

ALMEIDA-ANACleto, D.; MARCHINI, L. C. Composição físico-química de amostras de méis de Apis mellifera $\mathrm{L}$. provenientes do cerrado paulista. Boletim de Indústria animal, Nova Odessa, v. 61, n. 2, p. 161-172, abr./ maio 2004.

ARAÚJO, D. R. de; SILVA, R. H. D. da; SOUSA, J. dos S. Avaliação da qualidade físico-química do mel comercializado na cidade de Crato, CE. Revista de Biologia e Ciência da Terra, Campina Grande, v. 6, n. 1, jun. 2006. 
ARRUDA, C. M. F. de; MARCHINI, L. C.; MORETI, A. C. de C. C.; OTSUK, I. P.; SODRÉ, G. da S. Características físico-químicas de méis da Chapada do Araripe/ Santana do Cariri, Ceará. Acta Scientiarum Animal Sciences, Maringá, v. 27, n. 1, p. 171-176, jan./mar. 2005.

ASSOCIATION OF OFFICIALANALYTICAL COUNCIL. Official methods of analysis. 2. ed. Washington, DC, 1990. $1018 \mathrm{p}$.

ATAGO. Refratômetro para mel. Abelhas, v. 31, n. 362/363, p. 9, 11-12, 41, 44, 1988.

AZEREDO, M. A. A.; AZEREDO, L. da C. Características físico-químicas dos méis do município de São Fidélis-RJ. Ciência e Tecnologia de Alimentos, Campinas, v. 19, n. 1, p. 3-7, 1999.

BALDI-CORONEL, B.; DALL'OGLLIO, A. M.; LEZCANO, S. Caracterización físico químico de las mieles de la Provincia de Entre Rios. Alimentación Latinoamericana, v. 39, p. 39-44, 1993.

BARTH, M. O. Análise microscópica de algumas amostras de mel: 1 pólen dominante. Anais da Academia Brasileira de Ciências, Rio de Janeiro, v. 42, n. 2, p. 351-366, 1970a.

BARTH, M. O. Análise microscópica de algumas amostras de mel: 2 pólen acessório. Anais da Academia Brasileira de Ciências, Rio de Janeiro, v. 42, n. 3, p. 571-590, 1970b.

BARTH, M. O. Análise microscópica de algumas amostras de mel: 3 pólen isolado. Anais da Academia Brasileira de Ciências, Rio de Janeiro, v. 42, n. 4, p. 748-772, 1970c.

BARTH, M. O. O pólen no mel brasileiro. Rio de Janeiro: Luxor, 1989. 152 p.

BOGDANOV, S.; MARTIN, P.; LULLMANN, C. Harmonized methods of the european honey commission. Apidologie, Issue, p. 1-59, 1997. Supplement.

BOLETIN OFICIAL ESPAÑOL. Orden de 12 de junio de 1986, de la Presidencia del Gobierno por la que se aprueban los métodos oficiales de analisis para la miel. Madrid, 1986.

BRASIL. Ministério da Agricultura. Instrução normativa n. 11 , de 20 de outubro de 2000. Regulamento técnico de identidade e qualidade do mel. Diário Oficial, Brasília, p. 16-17, 20 out. 2000 . Seção I.
BUSSAB. W. O.; ANDRADE, D. F.; MYAZAKY, E. S. Introdução a análise de agrupamento. In: SIMPÓSIO NACIONAL DE PROBABILIDADE E ESTATÍSTICA, 9., 1990, São Paulo. Anais... São Paulo: IME/USP, 1990. $105 \mathrm{p}$.

CAMPOS, G. Melato no mel e sua determinação através de diferentes metodologias. 1989. $178 \mathrm{f}$. Tese (Doutorado em Ciência Animal) - Universidade Federal de Minas Gerais, Belo Horizonte, 1989.

CANO, C. B.; FELSNER, M. L.; MATTOS, J. R.; BRUNS, R. E.; WATANABE, H. M.; MURADIAN, L. B. A. Comparison of methods for determining moisture content of citrus and eucaplyptus brazilian honeys by refractometry. Journal of Food Composition Analysis, v. 14, p. 101-109, 2001.

CANO, C. B.; ZAMBONI, C. Q.; ALVES, H. I.; SPITERI, N.; ATUI, M. B.; SANTOS, M. C. dos; JORGE, L. I. F.; PEREIRA, U.; RODRIGUES, R. M. M. Mel: fraudes e condições sanitárias. Revista do Instituto Adolfo Lutz, São Paulo, v. 52, p. 1-4, 1992.

COOPERATIVA DE PRODUTORES DE CANA, AÇÚCAR E ÁLCOOL DO ESTADO DE SÃO PAULO. Métodos analíticos. In: Manual de controle químico da fabricação de açúcar. Piracicaba: Copersucar, 1987. p. $1-51$.

COSTA, L. S. M.; ALBUQUERQUE, M. L. S.; TURGO, L. C.; QUINTEIRO, L.; BARTH, O. M.; RIBEIRO, M.; MARIA, C. A. B. Determination of non-volatile compounds of different botanical origin brazilian honeys. Food Chemistry, London, v. 65, p. 347-352, 1999.

DAYRELL, I. O.; VITAL, N. C. Comparação entre dois métodos oficiais para determinação de hidroximetilfurfural (HMF) em mel brasileiro. Ciência e Tecnologia de Alimentos, Campinas, v. 1, n. 1, p. 137-141, 1991.

ERDTMAN, G. Pollen morphology and plant taxonomy: angiosperms. Stockholm: Almqvist \& Wiksell, 1952. 539 p.

EVANGELISTA-RODRIGUES, A.; SILVA, E. M. S.; BESERRA, E. M. F.; RODRIGUES, M. L. Análise fpisicoquímica dos mes de abelhas Apis mellifera e Melipona scutellaris produzidos em regiões distintas no Estado da Paraíba. Ciência Rural, Santa Maria, v. 35, n. 5, p. 11661171, 2005. 
GOMEZ, M. E. M.; HERNANDEZ, E. G.; GOMEZ, J. Y. M.; MARIN, J. L. M. Physicochemical analysis of Spanish commercial Eucaliptus honeys. Journal of Apicultural Research, v. 32, n. 3/4, p. 121-126, 1993.

JOLIFFE, I. T. Discarding variables in a principal component analysis: I real date. Applied Statistics, v. 21, n. 1, p. 21-31, 1973.

LOUVEAUX, J.; MAURIZIO, A.; VORWOHL, G. Methods of melissopalinology. Bee World, Bucks, v. 59, n. 4, p. 139$157,1978$.

MARCHINI, L. C.; MORETI, A. C. de C. C.; OTSUK, I. P. Análise de agrupamento, com base na composição físicoquímica de amostras de méis produzidos por Apis mellifera L. no Estado de São Paulo. Ciência e Tecnologia de Alimentos, Campinas, v. 25, n. 1, p. 8-17, 2005.

MARCHINI, L. C.; SODRÉ, G. S.; MORETI, A. C. de C. C. Mel brasileiro: composição e normas. Ribeirão Preto: A.S. Pinto, 2004a. 111 p.

MARCHINI, L. C.; SODRÉ, G. S.; MORETI, A. C. de C. C.; OTSUK, I. P. Composição físico-química de amostras de méis de Apis mellifera L. do Estado de Tocantins, Brasil. Boletim de Indústria Animal, Nova Odessa, v. 61, n. 2, p. 1001-114, 2004b.

MARDIA, L. V.; KENI, J. T.; BIBBY, J. M. Multivariate analysis. London: Academic, 1979. 521 p.

MORAES, R. M. de; TEIXEIRA, E. W. Análise de mel: manual técnico. Pindamonhangaba: [s.n.], 1998. 41 p.

MORETI, A. C. de C. C.; SODRE, G. S.; MARCHINI, L. C.; CARVALHO, C. A. L. Cor de amostras de méis de Apis mellifera $\mathrm{L}$. de diferentes estados brasileiros. Boletim de Indústria Animal, Nova Odessa, v. 63, n. 3, p. 159-164, 2006.

PREGNOLATO, W.; PREGNOLATO, N. P. (Coords.). Normas analíticas do Instituto Adolfo Lutz. 3. ed. São Paulo: Instituto Adolfo Lutz, 1985. v. 1, 533 p.

SEEMANN, P.; NEIRA, M. Tecnología de la producción apícola. Valdivia: Universidad Austral de Chile/Facultad de Ciencias Agrarias Empaste, 1988. 202 p.
SILVA, C. L.; QUEIROZ, A. J. M.; FIGUEIRÊDO, R. M. F. Caracterização físico-química de méis produzidos no Estado do Piauí para diferentes floradas. Revista Brasileira de Engenharia Agrícola e Ambiental, Campina Grande, v. 8, n. 2/3, p. 260-265, 2004.

SILVA, D. J.; QUEIROZ, A. C. Determinação do nitrogênio total e da proteína bruta. In: Análise de alimentos: métodos químicos e biológicos. 3. ed. Viçosa: UFV, 2002. p. 57-75.

SODRÉ, G. S.; MARCHINI, L. C.; CARVALHO, C. A. L. Características físico-químicas de amostras de méis de abelha Apis mellifera da região litoral norte do Estado da Bahia. Revista de Agricultura, v. 77, n. 2, p. 243-256, 2002.

SODRÉ, G. S.; MARCHINI, L. C.; MORETI, A. C. de C. C.; CARVALHO, C. A. L. Análises multivariadas com base nas características físico-químicas de amostras de méis de Apis mellifera L. (Hymenoptera: Apidae) da região litoral norte da Bahia. Archivo Latinoamericano de Produção Animal, v. 11, n. 3, p. 129-137, 2003.

SODRÉ, G. S.; MARCHINI, L. C.; ZUCCHI, O. L. A. D.; NASCIMENTO FILHO, V. F.; MORETI, A. C. de C. C.; OTSUK, I. P. Minerais encontrados em amostras de méis de Apis mellifera Africanizada (Hymenoptera: Apidae) provenientes de alguns municípios do Estado do Ceará. Boletim de Indústria animal, Nova Odessa, v. 62, n. 1, p. 918, 2005.

SPORNS, P.; PLHAK, L.; FRIEDRICH, J. Alberta honey composition. Food Research International, Barking, v. 25, n. 2, p. 93-100, 1992.

VIDAL, R.; FREGOSI, E. V. de. Mel: características, análises físico-químicas, adulterações e transformações. Barretos: Instituto Tecnológico Científico "Roberto Rios", 1984. 95 p.

VIEIRA, G. H. C.; MARCHINI, L. C.; DALASTRA, C. Caracterização físico-química de méis produzidos por Apis mellifera L. (Hymenoptera: apidae) em área de cerrado no município de Cassilândia, MS. Boletim de Indústria Animal, Nova Odessa, v. 62, n. 3, p. 203-214, 2005.

VILHENA, F.; ALMEIDA-MURADIAN, L. B. Análises físico-químicas de méis de São Paulo. Mensagem Doce, v. 53, p. 17-19, 1999. 